$10-1-1986$

\title{
Multicritical Points in an Ising Random-Field Model
}

\author{
Miron Kaufman \\ Cleveland State University, m.kaufman@csuohio.edu \\ Philip E. Klunzinger \\ Cleveland State University
}

A. Khurana

Princeton University

Follow this and additional works at: https://engagedscholarship.csuohio.edu/sciphysics_facpub

Part of the Physics Commons

How does access to this work benefit you? Let us know!

Publisher's Statement

Copyright 1986 American Physical Society. Available on publisher's site at http://link.aps.org/ doi/10.1103/PhysRevB.34.4766.

Original Citation

Kaufman, Miron, Philip E. Klunzinger, and A. Khurana. "Multicritical Points in an Ising Random-Field Model." Physical Review B 34 (1986): 4766-4770.

\section{Repository Citation}

Kaufman, Miron; Klunzinger, Philip E.; and Khurana, A., "Multicritical Points in an Ising Random-Field Model" (1986). Physics Faculty Publications. 100.

https://engagedscholarship.csuohio.edu/sciphysics_facpub/100

This Article is brought to you for free and open access by the Physics Department at EngagedScholarship@CSU. It has been accepted for inclusion in Physics Faculty Publications by an authorized administrator of EngagedScholarship@CSU. For more information, please contact library.es@csuohio.edu. 


\title{
Multicritical points in an Ising random-field model
}

\author{
Miron Kaufman and Philip E. Klunzinger \\ Department of Physics, Cleveland State University, Cleveland, Ohio 44115 \\ A. Khurana \\ Department of Physics, Princeton University, Princeton, New Jersey 08544
}

(Received 27 May 1986)

\begin{abstract}
The phase diagram of the mean-field Ising model in a random field obeying a symmetric threepeak distribution is determined. This distribution is relevant to diluted antiferromagnets in a uniform magnetic field. The phase diagram includes a fourth-order point, tricritical points, ordered critical points, critical end points, and a double critical end point. An ordered phase persists for arbitrarily large random fields at low temperatures.
\end{abstract}

\section{INTRODUCTION}

Experimental $^{1}$ and theoretical ${ }^{2,3}$ studies of Ising systems subjected to random magnetic fields have concentrated mainly on the question of what is the lower critical dimension. The consensus opinion is that the lowest spatial dimension (lower critical dimension) above which long-range order can be established, is two. ${ }^{2}$ However, strong hysteretic effects ${ }^{1}$ prevent or make it difficult to observe the ordered phase in equilibrium. Still unresolved is the issue ${ }^{4}$ of what crossover is actually observed in some experiments, whether random field to random exchange or random field to classical criticality.

This paper addresses the effect of different field distributions on the topology of the equilibrium phase diagram. Most of the theoretical work has focused on the Gaussian ${ }^{5}$ and two-peak ${ }^{6}$ distributions. We consider here random fields which can take three values $+h_{0},-h_{0}$, or 0 , with probabilities $p / 2, p / 2$, or $1-p$, respectively. This threepeak symmetric distribution is relevant ${ }^{1}$ to diluted antiferromagnets, such as $\mathrm{Fe}_{x} \mathrm{Zn}_{1-x} \mathrm{~F}_{2}$, in a uniform field $h_{0}$. In the Zeeman Hamiltonian ${ }^{1}$ the field conjugated to the antiferromagnetic order parameter (one-half of the difference between neighboring spins on opposite sublattices) takes three values: $h_{0},-h_{0}$, and 0 with probabilities $p / 2=x(1-x), p / 2=x(1-x)$, and $1-p=x^{2}+(1-x)^{2}$, where $1-x$ is the concentration of nonmagnetic impurities. This distribution has also been considered by Mattis, ${ }^{7}$ who calls it the trimodal distribution. Grinstein and Mukamel ${ }^{8}$ have studied the case: $h_{0} \rightarrow \infty$ for a onedimensional system.

We determine the complete phase diagram of the equivalent-neighbor (mean-field) Ising model with random fields obeying the three-peak symmetric distribution. We find a variety of multicritical points: fourth-order, tricritical, ordered critical, critical end, and double critical end. With the exception of the tricritical point, none of the others have been detected in a previous study ${ }^{7}$ of the model.

For $p<1$, an ordered (ferromagnetic) phase exists for arbitrarily large values of the random-field strength, $h_{0}$. In the context of the equivalent-neighbor model, this out- come is a consequence of the fact that a fraction $1-p$ of the spins are subjected to no magnetic field, and are sufficiently interconnected by the equivalent-neighbor bonds to order at low temperatures. We believe that the ordered phase will also be formed on a finite-dimensional lattice, provided $p$ is sufficiently small. Indeed, if $p<1-p_{c}$ (where $p_{c}$ is the site-percolation threshold) there is a finite probability for the formation of an infinite cluster of spins subjected to zero magnetic field. If this cluster is not treelike, we expect the ordered phase, which forms at zero temperature to persist at low but finite temperatures, for arbitrarily large $h_{0}$.

For $p=\frac{1}{3}$, the three-peak distribution was argued ${ }^{7}$ to be a good approximation of the Gaussian distribution, because the low-order momenta $\left\langle h^{2}\right\rangle$ and $\left\langle h^{4}\right\rangle$ are the same, respectively, for both distributions. Indeed, no tricritical point occurs for $p=\frac{1}{3}$, which is also true for the Gaussian distribution. 5 On the other hand, the Gaussian distribution phase diagram has no ordered phase for large random fields, while the three-peak distribution exhibits an ordered phase for arbitrarily large $h_{0}$ at low temperatures. Therefore, different field distributions will result, in general, in topologically different phase diagrams. The matching of the low-order momenta does not ensure the equivalence of the phase diagrams except at low values of the random-field strength.

The remainder of this article contains a description of the model and its solution, in Sec. II, and our concluding remarks in Sec. III.

\section{MODEL AND SOLUTION}

In the equivalent-neighbor Ising model, each pair of the $N$ spins $\sigma_{i}= \pm 1$ is connected by a bond of strength $1 / N$. Each spin is subjected to a random magnetic field $h$ according to the following distribution:

$$
\begin{aligned}
P(h)= & \frac{1}{2} p\left[\delta\left(h-h_{0}\right)+\delta\left(h+h_{0}\right)\right] \\
& +(1-p) \delta(h),
\end{aligned}
$$

where $0 \leq p \leq 1, h_{0} \geq 0$, and $\delta$ is the Dirac delta function. The energy $\mathscr{H}$ associated with the $N$ spins is 


$$
-\mathscr{H}=\frac{1}{2 N} \sum_{i j} \sigma_{i} \sigma_{j}+\sum_{i} h_{i} \sigma_{i}
$$

The quenched averaged free energy is

$$
f=-k_{B} T\left\langle\frac{1}{N} \ln Z\right\rangle,
$$

where $Z$ is the partition function for a given configuration of the magnetic fields. In the thermodynamic limit: ${ }^{5}$

$$
f=k_{B} T \min _{m} \psi(m),
$$

where

$$
\psi(m)=\frac{1}{2} J m^{2}-\langle\ln 2 \cosh J(m+h)\rangle .
$$

In Eq. (5): $J=1 / k_{B} T, m$ is the average site magnetization, and \langle\rangle denotes an average over the magnetic fields, using the distribution of Eq. (1).

The detailed analysis of this model involves a numerical solution of Eqs. (4) and (5), which is presented in subsection $\mathrm{C}$ below. Certain features of the phase diagram are determined analytically; in subsection $\mathbf{A}$, zero temperature, and in subsection B, multicritical points.

\section{A. Zero temperature}

At zero temperature, $T=0$ or $J=\infty$, Eq. (5) reduces to

$$
\frac{\psi}{J}=\frac{1}{2} m^{2}-\langle|m+h|\rangle \text {. }
$$

The values of $m$ which minimize $\psi$ are the following: for small $h_{0}, m= \pm 1$, and for large $h_{0}, m= \pm(1-p)$. A first-order transition between the two ordered phases occurs at $h_{0}=1-p / 2$. The transition point, counting both positive and negative $m$ 's, is actually a point where four phases coexist.

The physical origin of the two ordered phases is as follows. For small $h_{0}$ the bonds are strong enough to allow all spins to order, i.e., $m= \pm 1$. For large $h_{0}$, a fraction $p$ of the spins randomly points up and down, aligned with the local fields, while a fraction $1-p$ of the spins, subjected to no field, is in order, i.e., $m= \pm(1-p)$. The essential feature is that in the latter group the spins are interconnected by the equivalent-neighbor bonds, forming an infinite cluster which can order at low temperatures.

This condition should also be satisfied for a finite dimensional lattice, if $1-p$ is larger than $p_{c}$, the sitepercolation threshold. Hence, for $p<1-p_{c}$ an infinite cluster is formed. Provided this cluster has loops, i.e., it is not treelike, the spins on the percolating cluster will order at low temperatures even for large fields. In the onedimensional version ${ }^{8}$ of the model with $h_{0} \rightarrow \infty, m$ is zero for all $p>0$, which is consistent with our argument because the one-dimensional threshold is $p_{c}=1$.

\section{B. Multicritical points}

Close to a continuous transition between ordered and disordered phases, $m$ is small, and we can expand $\psi$ in powers of $m$ :

$$
\psi(m)-\psi(0)=a_{2} m^{2}+a_{4} m^{4}+a_{6} m^{6}+a_{8} m^{8} .
$$

The $a$ coefficients are the following:

$$
\begin{aligned}
& a_{2}=\frac{1}{2} J^{2}\left(\frac{1}{J}-1+p u\right), \\
& a_{4}=\frac{1}{12} J^{4}\left(1-4 p u+3 p u^{2}\right), \\
& a_{6}=\frac{1}{90} J^{6}\left(-2+17 p u-30 p u^{2}+15 p u^{3}\right), \\
& a_{8}=\frac{1}{2520} J^{8}\left(17-248 p u+756 p u^{2}-840 p u^{3}+315 p u^{4}\right),
\end{aligned}
$$

where $J=1 / k_{B} T$ and $u=\tanh ^{2}(J h)$.

Following Griffiths, ${ }^{9}$ we use the following symbols to denote the various entities on the phase diagram: $A-$ one-phase point (disordered phase); $A^{2}$-two-phase point (ordered phase); $A^{3}$-three-phase point (coexistence of ordered $A^{2}$ and disordered $A$ phases); $A^{4}$-four-phase point (coexistence of two ordered $A^{2}$ phases); $B-$ critical point; $B A^{2}$-critical end point (coexistence of critical $B$ and ordered $A^{2}$ phases); $B^{2}$-isolated critical point in the midst of an ordered phase (coexistence of two critical $B$ phases), which we shall call ordered critical point; $C$-tricritical point; and $D$-fourth-order point.

The fourth-order point $D$ occurs at $a_{2}=a_{4}=a_{6}=0$, and $a_{8}>0$. From Eq. (8) we compute the coordinates of this point: $p=\frac{25}{33} \cong 0.76, k_{B} T=\frac{6}{11} \cong 0.55$, and $h_{0} \cong 0.56$. This point lies at the intersection ${ }^{10}$ of a line of tricritical $^{11,12}$ points $C$, a line of ordered critical points $B^{2}$, and a line of critical end points $B A^{2}$. It can therefore be viewed as the last tricritical point, i.e., for $p<0.76$ there are no tricritical points. Mattis ${ }^{7}$ has estimated the location of this point by first determining the line of tricritical points from $a_{2}=0$ and $d h_{0} / d T=0$, where

$$
k_{B} T=1-p \tanh ^{2}\left(h_{0} / k_{B} T\right)
$$

is the critical line; then he looked for the "vestigial" tricritical point (the $D$ point). His estimate is $p=0.75$, $k_{B} T=0.44$, and $h_{0}=0.57$. The discrepancy between Mattis's and our results is small for $p$ and $h_{0}$, less than $2 \%$, but rather substantial for the temperature, about $20 \%$. The source of this discrepancy is the fact that the line of tricritical points is exactly located at $a_{2}=a_{4}=0$ and $a_{6}>0$, while Mattis's requirement $d h_{0} / d T=0$ is not a necessary condition for a tricritical point to occur. The latter provides, however, a lower bound on the correct tricritical temperature, and an upper bound on the correct tricritical $h_{0}$, if no reentrance of the disordered phase occurs (i.e., $d h_{0} / d T$ always negative). The $20 \%$ discrepancy on the temperature estimate indicates that Mattis's method to determine tricritical coordinates, first used in the high-temperature analyses ${ }^{13}$ of random-field systems, is not always accurate. To obtain another estimate of accuracy, we looked at the tricritical point at $p=1$, exactly located ${ }^{6}$ at $k_{B} T=0.67$ and $h_{0}=0.44$. Mattis's approximation locates the tricritical point at $k_{B} T=0.58$ and $h_{0}=0.45$. The percentage errors are $13 \%$ for temperature and $2 \%$ for $h_{0}$.

The ordered critical point $B^{2}$, terminates a line of firstorder transition points $A^{4}$, in the midst of the ordered phase $A^{2}$. In the $\left(a_{2}, a_{4}, a_{6}\right)$ space the $B^{2}$ points form a line which, close to the fourth-order point, is

$$
\frac{a_{2}}{a_{8}}=\frac{1}{16}\left(\frac{a_{6}}{a_{8}}\right)^{3}, \frac{a_{4}}{a_{8}}=\frac{3}{8}\left(\frac{a_{6}}{a_{8}}\right)^{2}, a_{6}<0, a_{8}>0 \text {. }
$$




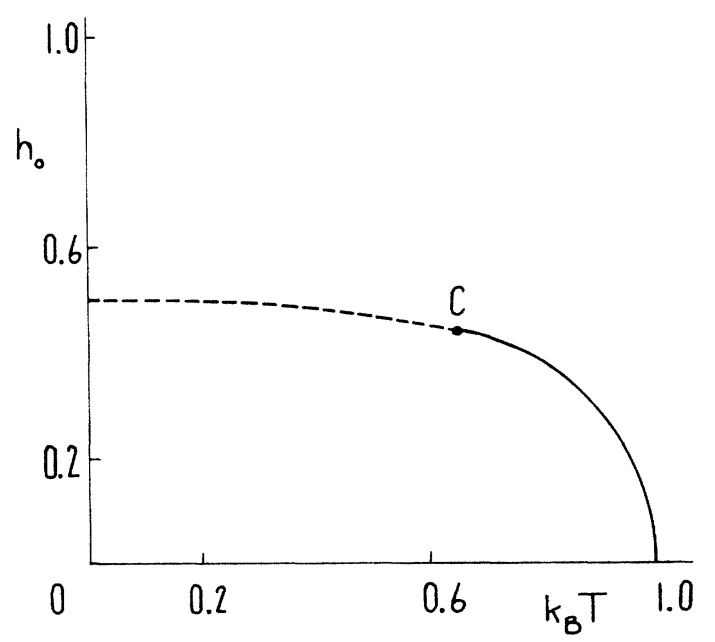

FIG. 1. Phase diagram for $p=1$ : solid line, critical points; dashed line, first-order transitions. A tricritical point $C$ also occurs.

The critical end point $B A^{2}$ occurs at the intersection of a line of critical points $B$ and a line of first-order transition points $A^{3}$ and $A^{4}$. Close to the fourth-order point, $B A^{2}$ coordinates are the following:

$$
a_{2}=0, \quad \frac{a_{4}}{a_{8}}=\frac{1}{4}\left(\frac{a_{6}}{a_{8}}\right)^{2}, \quad a_{6}<0, a_{8}>0
$$

The critical points $B$ form a surface in the $\left(a_{2}, a_{4}, a_{6}\right)$ space, or in the $\left(T, h_{0}, p\right)$ space which, when not preempted by first-order transitions, is given by

$$
a_{2}=0 \text { or } k_{B} T=1-p \tanh ^{2}\left(h_{0} / K_{B} T\right) .
$$

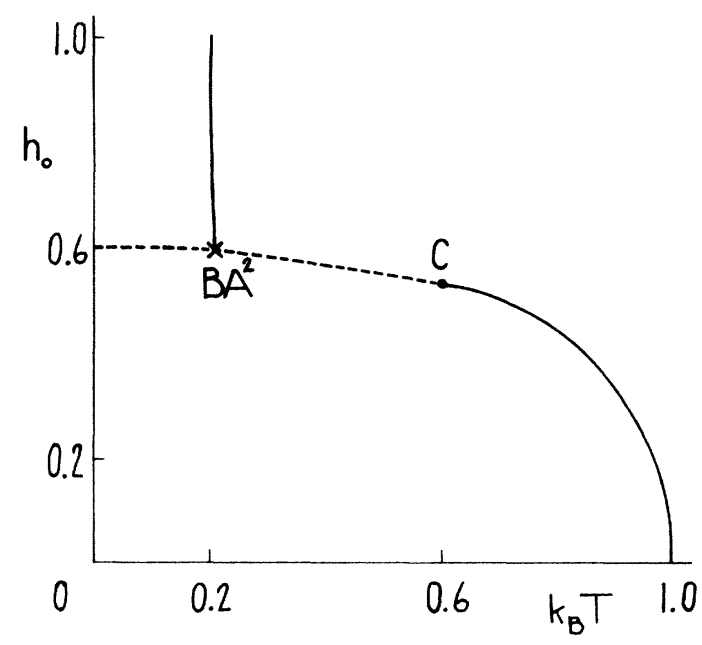

FIG. 2. Phase diagram for $p=0.8$ : solid line, critical points; dashed line, first-order transitions. A critical end point $B A^{2}$ and a tricritical point $C$ also occur.

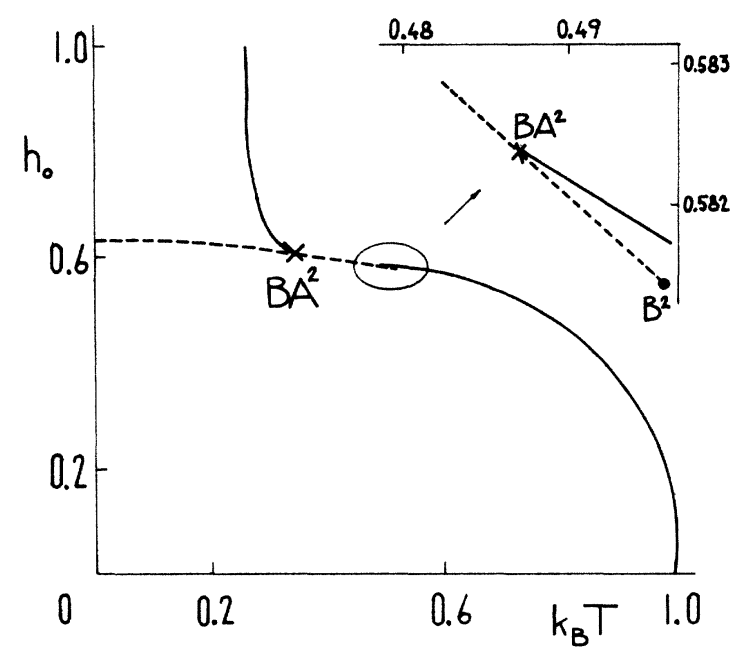

FIG. 3. Phase diagram for $p=0.74$ : solid line, critical points; dashed line, first-order transitions. Two critical end points $B A^{2}$ and an ordered critical point $B^{2}$ also occur. The inset shows a closer view of the region in the oval.

\section{Phase diagram}

We determine numerically the phase diagrams in the plane $T, h_{0}$ for different values of $p$. There are two special $p$ values: $p=\frac{25}{33} \cong 0.76$ and $p \cong 0.73$, which divide the interval $[0,1]$ in three subintervals corresponding to three topologically different types of phase diagrams.

For $p=1$, we obtain Aharony's ${ }^{6}$ phase diagram, Fig. 1 , with a tricritical point at $k_{B} T=0.67$ and $h_{0}=0.44$. A new ordered phase appears for any $p<1$, at large $h_{0}$. On this phase the magnetization is smaller than that of the ordered phase at weak $h_{0}$. The two ordered phases are separated, at low temperatures, by a first-order line of $A^{4}$ points, starting at $h_{0}=1-p / 2$, at $T=0$. The small- $m$ ordered phase terminates at a line of critical points which,

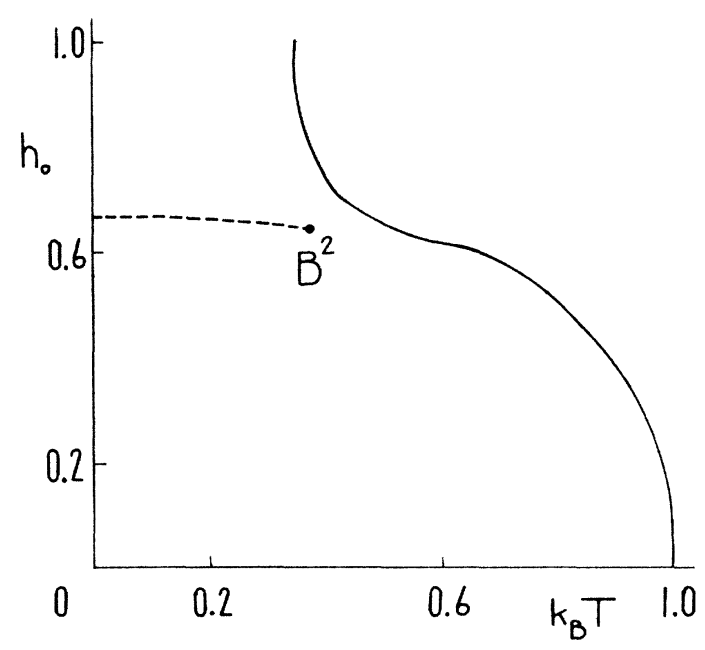

FIG. 4. Phase diagram for $p=0.67$ : solid line, critical points; dashed line, first-order transitions. An ordered critical point $B^{2}$ also occurs. 


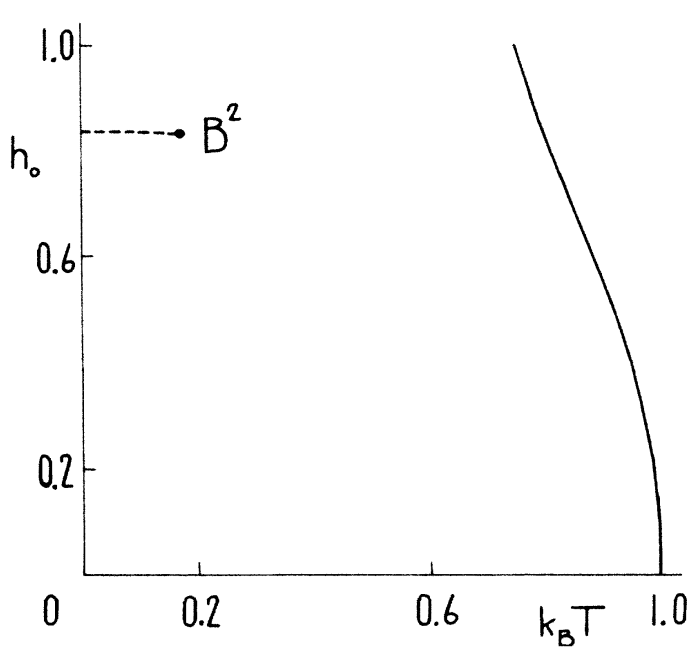

FIG. 5. Phase diagram for $p=0.33$ : solid line, critical points; dashed line, first-order transitions. An ordered critical point $B^{2}$ also occurs. Contrary to a suggestion in Ref. 7, this phase diagram qualitatively differs from the phase diagram for the Gaussian distribution.

for $h_{0} \rightarrow \infty$, is at $k_{B} T=1-p$. As $p$ is lowered, the tricritical point persists for $p>\frac{25}{33} \cong 0.76$, Fig. 2. The "vestigial" tricritical point, at $p=\frac{25}{33}$, is the fourth-order point occurring at $k_{B} T=\frac{6}{11} \cong 0.55$ and $h_{0} \cong 0.56$. A critical end point, $B A^{2}$, occurs at the intersection of a critical line terminating the small $m$ phase and the first-order line separating the small $m$ and the disordered phases from the large $m$ phase.

For $0.73<p<0.76$, a new type of phase diagram appears (Fig. 3), in which the tricritical point is replaced by an ordered critical point $B^{2}$ and a new critical end point $B A^{2}$, i.e., there are now two critical end points. As $p \rightarrow 0.73$, the two critical end points coalesce. This coalescing point is not a new multicritical point. Viewed in the three-dimensional parameter space $\left(T, h_{0}, p\right)$ it lies on a smooth line of $B A^{2}$ points, at an extremum. It could be called a double critical end point, by analogy with the double critical point (see Appendix A of Ref. 11) which plays a similar role on a line of critical points.

For $0<p<0.73$, we obtain a third type of phase diagram (Figs. 4 and 5), with the critical line uninterrupted from $h_{0}=0, k_{B} T=1$, to $h_{0} \rightarrow \infty, k_{B} T=1-p$. In the ordered phase there is a first-order line of $A^{4}$ points ending at an ordered critical point $B^{2}$. This first-order line shrinks to zero as $p$ goes to zero. At $p=0$ the model reduces to the pure Ising model in zero magnetic field, and $h_{0}$ is a redundant variable which does not affect the free energy. The phase diagram (Fig. 6) has a critical line at $k_{B} T=1$, for any $h_{0}$.

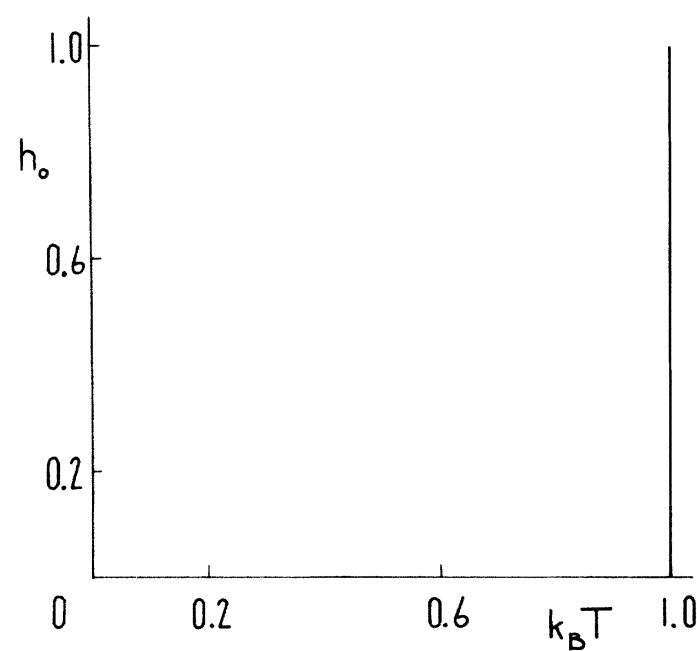

FIG. 6. Phase diagram for $p=0$. The vertical solid line is a line of critical points.

\section{CONCLUDING REMARKS}

We have determined the complete phase diagram of the equivalent-neighbor (mean-field) Ising model in a random field obeying a three-peak symmetric distribution. The main conclusion of our study is that the topology of the phase diagram depends strongly on the particular random-field distribution used. Previously studied phase diagrams for the Gaussian and two-peak distributions resemble each other topologically, even though the order of the transition differs at low temperatures. In particular, for both distributions, there is no ordered phase for large random fields at low temperatures. The phase diagram for the symmetric three-peak distribution is qualitatively different: the ordered phase persists for arbitrarily large random fields at low temperatures. We believe this ordered phase occurs for realistic short-range interactions in finite dimensions.

Diluted antiferromagnets, e.g., $\mathrm{Fe}_{x} \mathrm{Zn}_{1-x} \mathrm{~F}_{2}$, in a uniform field are realizations of the random-field Ising model with random fields obeying a three-peak symmetric distribution with $p=2 x(1-x)$ (see Sec. I). In spite of differences between the model and its experimental realizations, such as the random character of the exchange interactions in the experimental systems, it is conceivable that an ordered phase will form even for large magnetic fields if $1-p$ is larger than the site-percolation threshold $p_{c}$. In terms of the magnetic concentration $x$ this means $x<\left[1-\left(2 p_{c}-1\right)^{1 / 2}\right] / 2$ and $x>\left[\left(1+\left(2 p_{c}-1\right)^{1 / 2}\right] / 2\right.$ if $p_{c}$ is larger than $\frac{1}{2}$. If $p_{c}$ is smaller than $\frac{1}{2}$ the long-range order should be established at large fields for any concentration $x$. Since $p=2 x(1-x) \leq 0.5$, our computations suggest the absence of a tricritical point for diluted antiferromagnets in a uniform field.
${ }^{1}$ R. J. Birgeneau, Y. Shapira, G. Shirane, R. A. Cowley, and H. Yoshizawa, Physica B 137, 83 (1986), and references therein; Po-zen Wong, S. von Molnar, and P. Dimon, J. Appl. Phys. 53, 7954 (1982).

${ }^{2}$ Y. Imry and S.-K. Ma, Phys. Rev. Lett. 35, 1399 (1975); J. Z. Imbrie, ibid. 53, 1747 (1984).
${ }^{3}$ A. Aharony, Y. Imry, and S.-K. Ma, Phys. Rev. Lett. 37, 1364, (1976).

${ }^{4}$ M. Kaufman and M. Kardar, Phys. Rev. B 31, 2913 (1985); M. Kaufman, Superlattices and Microstructures 1, 511 (1985), and references therein.

${ }^{5}$ T. Schneider and E. Pytte, Phys. Rev. B 15, 1519 (1977). 
${ }^{6}$ A. Aharony, Phys. Rev. B 18, 3318 (1978).

${ }^{7}$ D. C. Mattis, Phys. Rev. Lett. 55, 3009 (1985).

${ }^{8}$ G. Grinstein and D. Mukamel, Phys. Rev. B 27, 4503 (1983).

${ }^{9}$ R. B. Griffiths, Phys. Rev. B 12, 345 (1975).

${ }^{10} \mathrm{~A}$ similar topology was observed in the two-dimensional BEG model; A. N. Berker and M. Wortis, Phys. Rev. B 14, 4946
(1976).

${ }^{11}$ M. Kaufman, R. B. Griffiths, J. M. Yeomans, and M. E. Fisher, Phys. Rev. B 23, 3448 (1981).

12S. Galam, Phys. Rev. B 31, 7274 (1985).

${ }^{13}$ A. Houghton, A. Khurana, and F. J. Seco, Phys. Rev. B 34, 1700 (1986); Phys. Rev. Lett. 55, 856 (1985). 